\title{
Association of obstructive sleep apnea/hypopnea syndrome with glaucomatous optic neuropathy and ocular blood flow
}

\author{
GEORGIOS DALIANIS $^{1}$, ALEXANDRA TRIVLI ${ }^{1}$, ALIKI LIMNOPOULOU ${ }^{2}$, ANDREAS STAVRAKIS ${ }^{3}$, \\ CHRYSSA TERZIDOU $^{1}$, EFSTATHIOS T. DETORAKIS ${ }^{4}$, VASSILIOS KOZOBOLIS $^{5,6}$ and GEORGIOS LABIRIS ${ }^{6}$ \\ ${ }^{1}$ Department of Ophthalmology, Konstantopouleio-Patission General Hospital, Athens 14233; \\ ${ }^{2}$ Department of Ophthalmology, Public Health Center, Athens 10679; ${ }^{3}$ Department of Sleep Apnea, \\ Konstantopouleio-Patission General Hospital, Athens 14233; ${ }^{4}$ Department of Ophthalmology, \\ University Hospital of Heraklion, Heraklion 71110; ${ }^{5}$ Eye Institute Thrace, Alexandroupolis 68131; \\ ${ }^{6}$ Department of Ophthalmology, University Hospital of Alexandroupolis, Alexandroupolis 68100, Greece
}

Received November 16, 2020; Accepted March 18, 2021

DOI: $10.3892 / \mathrm{etm} .2021 .10089$

\begin{abstract}
The present study aimed to investigate the prevalence of glaucomatous optic neuropathy in patients with obstructive sleep apnea/hypopnea syndrome (OSAHS). In total, 83 subjects (45 cases of severe OSAHS and 38 controls) underwent polysomnographic assessment and were evaluated for the severity of the disease using the Apnea-Hypopnea index. A detailed ophthalmologic exam was then performed, including measurement of the intraocular pressure (IOP) with a Goldmann applanation tonometer and Pascal dynamic contour tonometer (DCT), recording of the ocular pulse amplitude measured by the Pascal DCT, standard automated perimetry and retinal nerve fiber layer (RNFL) and ganglion cell complex (GCC) thickness evaluation using optical coherence tomography. Ocular blood flow was assessed using color Doppler imaging (CDI) and ophthalmic artery indices were evaluated, including peak systolic blood velocity, end diastolic blood velocity and resistivity index (RI). There was a significant difference in the mean IOP between controls $(11.03 \pm 3.85 \mathrm{mmHg})$ and cases of severe OSAHS $(18.06 \pm 3.39 \mathrm{mmHg})$ when the IOP was measured by DCT $(\mathrm{P}<0.0001)$, but not with the Goldmann applanation tonometer (IOP, $13.97 \pm 2.85 \mathrm{mmHg}$ for controls and $14.89 \pm 3.21 \mathrm{mmHg}$ for cases of severe OSAHS; $\mathrm{P}=0.0877)$. Significant negative correlations were observed between RNFL thickness and RI $(\mathrm{P}=0.0011)$ in cases of severe OSAHS, as well as between GCC thickness and RI $(\mathrm{P}<0.008)$ in all subjects. Furthermore, a negative correlation between RI
\end{abstract}

Correspondence to: Dr Alexandra Trivli, Department of Ophthalmology, Konstantopouleio-Patission General Hospital, 3-5 Agias Olgas, Nea Ionia, Athens 14233, Greece

E-mail: alextrivli@yahoo.com

Key words: obstructive sleep apnea/hypopnea syndrome, glaucomatous optic neuropathy, retinal nerve fiber layer, ganglion cell complex, resistivity index and RNFL thickness in severe cases of OSAHS suggested a hemodynamically induced vulnerability of RNFL in OSAHS. The correlation between RI and GCC thickness in all subjects suggested that impaired perfusion, more prominent in OSAHS, leads to structural changes. Therefore, cases of severe OSAHS should be monitored for changes in RNFL and GCC thickness, as well as CDI findings. Furthermore, patients with increased ophthalmic artery RIs should be monitored for changes in the GCC, regardless of the etiology of the RI increase.

\section{Introduction}

Obstructive sleep apnea/hypopnea syndrome (OSAHS) is the most common type of sleep respiratory disorder and is characterized by repetitive, complete or partial collapse of the pharyngeal airway during sleep, which leads to a reduction in oxygen desaturation and poor sleep quality (1). With an estimated prevalence of $4 \%$ in males and $2 \%$ in females, various risk factors have been implicated, including obesity, age, male sex and a thick neck diameter (1).

A number of sleeping disorders, including OSAHS, are associated with ocular conditions, such as floppy eyelid syndrome, keratoconus, retinal vascular congestion and bleeding, non-arteritic anterior ischemic optic neuropathy and glaucomatous optic neuropathy $(\mathrm{GON})$, and have been implicated in the impaired autoregulation of optic nerve head perfusion as a direct effect of hypoxia (2-5).

GON is a group of disorders characterized by progressive optic neuropathy and the degeneration of retinal ganglion cells, resulting in characteristic alteration (cupping) of the optic nerve head, as well as a pattern of visual field loss (6). The prevalence of glaucoma in the general population is $1-2 \%$ (7). Damage to the optic nerve may occur directly by the effect of elevated intraocular pressure (IOP) or due to impaired perfusion, and factors implicated in these processes include diabetes, obesity, cardiovascular disease, systemic hypertension or hypotension, vasospasms, autoregulatory defects and atherosclerosis, particularly in patients with normal IOP $(8,9)$. 
A possible association between OSAHS and GON has been the subject of various studies, which have proposed OSAHS as a risk factor for developing glaucoma and have reported an increased prevalence of OSAHS (ranging from 5.9-27\%) among patients with glaucoma and vice versa $(2,3,10-14)$. Therefore, the present study aimed to investigate this possible association and its underlying mechanisms.

\section{Patients and methods}

Patients. A total of 45 patients with newly diagnosed severe OSAHS, as determined by the Apnea Hypopnea Index (AHI) (1), were selected and 38 controls were matched with OSAHS cases for sex, age and body mass index. All subjects were prospectively, consecutively recruited from the Sleep Apnea Department of Konstantopouleio-Patission Hospital (Athens, Greece) between September 2016 and December 2017. A total of 58 male and 25 female patients aged $48-80$ years were recruited.

Inclusion and exclusion criteria. Patients with a history of ocular surgery (including laser treatments), ocular trauma, anterior or posterior segment disease, chronic steroid use, alcohol abuse, bronchial asthma, interstitial lung diseases, cerebrovascular disease, diabetes mellitus, heavy smoking (defined as >20 packs/year), systemic hypertension and atherosclerotic factors with a potential effect on blood flow were excluded from the study. With regard to the controls, additional ophthalmic criteria for inclusion in the study were as follows: IOP $<21 \mathrm{mmHg}$ (at three separate visits), no clinical evidence of GON (such as cupping, neuroretinal rim thinning or notching, disk hemorrhages, C/D asymmetry between eyes $>0.2$ ), normal anterior chamber angle on slit lamp gonioscopy, normal visual field tests and no previous history of glaucoma medication use.

Patient assessment. Subjects underwent polysomnography using a computerized system (SOMNOscreen Plus; Somnomedics $\mathrm{GmbH}$ ). This examination included the following: Electroencephalography, electrocardiography, electromyography, assessment of arterial blood oxygen saturation, snoring recording, nasobuccal airflow detection, chest wall and thoracic effort monitoring and body position recording. Factors analyzed included apnea, hypopnea, mean arterial blood oxygen saturation and snoring time. The number of apneic and hypopneic events per hour of sleep, indicated by the Respiratory Disturbance Index (RDI), was also documented (5). The severity of sleep apnea was graded according to the AHI. A score of $<5$ was considered normal, that of 6-15 was graded as mild, that of 16-30 was graded as moderate and that of $>30$ was considered as severe (15).

All patients underwent orbital color Doppler imaging (CDI; Logic E9; GE Healthcare). An 11-MHz linear surface probe was used and the measurements were performed with patients in the supine position, gazing upwards with closed eyelids. Peak systolic blood velocity (PSV) and end diastolic blood velocity (EDV) in the ophthalmic artery were calculated. The resistivity index (RI) was calculated using Pourcelot's formula (RI=PSV-EDV/PSV).
The detailed ophthalmological examination included recording of the best corrected visual acuity, slit lamp examination of the anterior segment, measurement of IOP with a Goldmann applanation tonometer (Haag-Streit AG) and Pascal DCT (Ziemer Ophthalmic Systems AG), recording of the ocular pulse amplitude (OPA) measured by a Pascal DCT, gonioscopic examination and fundus examination after mydriasis. Visual fields were evaluated using a Humphrey 740i field analyzer (Sita standard 24-2 strategy; Zeiss AG). Retinal nerve fiber layer (RNFL) thickness and ganglion cell complex (GCC) were examined via optical coherence tomography (OCT; Optovue, Inc.).

Statistical analysis. All statistical analysis was performed using the statistical software JMP Pro version 13 (SAS Institute, Inc.). Statistical analysis was performed using linear regression between various parameters based on controls and non-control sets.

All data were checked for normality of distribution using a Shapiro-Wilk test. For data determined to follow a normal distribution, comparison between the two groups was performed and P-values were determined by Student's t-test. For ordinal parameters not following a normal distribution, contingency analysis was used and P-values were determined using Fisher's exact test. In order to examine differences for nominal parameters that did not follow a normal distribution, the non-parametric Mann-Whitney U-test was used to determine P-values.

Linear regression was used for the estimation of significant predictors of the outcome variable, indicated by the magnitude and sign of the beta estimates. These regression estimates were used to explain the relationship between one dependent variable and one or more independent variables. Analysis results were presented in a scatterplot. $\mathrm{P}<0.05$ was considered to indicate a statistically significant difference.

\section{Results}

Patient information. Patient characteristics and ocular parameters are presented in Table I, while the results of the polysomnographic study and CDI parameters are presented in Table II. There was no statistical difference between male and female patients between the two groups with regards to sex $(\mathrm{P}=0.09)$ and age $(\mathrm{P}=0.21)$.

$I O P$. No statistically significant difference was identified in the mean IOP when measured with DCT between controls and OSAHS cases $(\mathrm{P}=0.88)$, as well as with a Goldmann applanation tonometer $(\mathrm{P}=0.17$; Table I). For the control group, a significant difference was observed in the measurement of IOP using either method $(\mathrm{P}=0.0003)$ and for cases of severe OSAHS, the difference in IOP was also significant $(\mathrm{P}<0.0001)$.

Other glaucoma parameters. When comparing the two groups, CCT $(\mathrm{P}=0.23)$, mean defect in visual field testing $(\mathrm{P}=0.19)$ and average GCC $(\mathrm{P}=0.55)$ exhibited no statistically significant difference. Similarly, average, superior and inferior RNFL values were not significantly different between controls and OSAHS cases (Table I). 
Table I. Demographic data and ocular parameters for the cohort.

\begin{tabular}{|c|c|c|c|}
\hline Item & Controls $(\mathrm{n}=38)$ & Severe OSAHS $(n=45)$ & P-value \\
\hline Sex (male/female) & $23 / 15$ & $35 / 10$ & $0.09^{\mathrm{a}}$ \\
\hline Age (years) & $59.8 \pm 8.3(57.1$ to 62.6$)$ & $62.1 \pm 7.6(59.8$ to 64.3$)$ & 0.21 \\
\hline IOP Goldmann (mmHg) & $14.0 \pm 2.6(13.0$ to 14.9$)$ & $14.9 \pm 3.2(13.9$ to 15.8$)$ & 0.17 \\
\hline IOP Pascal (mmHg) & $18.2 \pm 2.6(17.3$ to 19.0$)$ & $18.1 \pm 3.4(17.1$ to 19.1$)$ & 0.88 \\
\hline OPA Pascal (mmHg) & $2.6 \pm 0.9(2.3$ to 2.9$)$ & $2.7 \pm 0.9(2.5$ to 3.0$)$ & 0.59 \\
\hline $\mathrm{CCT}(\mu \mathrm{m})$ & $550.7 \pm 41.2(537$ to 564$)$ & $561.0 \pm 35.0(550$ to 571$)$ & 0.23 \\
\hline Visual field (MD) & $-1.0 \pm 2.0(-1.6$ to -0.3$)$ & $-1.6 \pm 2.1(-2.2$ to -0.9$)$ & 0.19 \\
\hline Average RNFL & $104.9 \pm 8.6(102.0$ to 108.0$)$ & $101.5 \pm 11.2(98.0$ to 105.0$)$ & 0.13 \\
\hline Superior RNFL & $103.4 \pm 9.6(100.3$ to 106.6$)$ & $99.16 \pm 11.79(95.6$ to 102.7$)$ & 0.07 \\
\hline Inferior RNFL & $106.16 \pm 9.10(103.2$ to 109.2$)$ & $103.80 \pm 12.39(100.1$ to 107.5$)$ & 0.32 \\
\hline Average GCC & $93.6 \pm 7.8(91.0$ to 96.1$)$ & $94.6 \pm 8.4(92.1$ to 97.2$)$ & 0.55 \\
\hline
\end{tabular}

aP-value determined by Fisher's exact test on contingency analysis. All other parameters followed a normal distribution according to the Shapiro-Wilk test for normality and P-values were determined using the t-test. Values are expressed as the mean \pm standard deviation (95\% CI) or n. OSAHS, obstructive sleep apnea-hypopnea syndrome; IOP, intraocular pressure; CCT, central corneal thickness; MD, mean defect; RNFL, retinal nerve fiber layer; GCC, ganglion cell complex; OPA, ocular pulse amplitude.

Table II. Results of polysomnographic study and color Doppler imaging parameters.

\begin{tabular}{lccc}
\hline Parameter & Controls & Severe OSAHS & P-value \\
\hline AHI & $4.0 \pm 3.0(3.0$ to 6.0$)$ & $37.4 \pm 15.4(32,8$ to 42,0$)$ & $<0.001^{\text {a,b }}$ \\
Saturated $\mathrm{O}_{2} \%$ & $94.5 \pm 1.0(92,2$ to 94,8$)$ & $93.8 \pm 1.5(93,3$ to 94,2$)$ & $0.02^{\mathrm{a}, \mathrm{b}}$ \\
RDI & $29.5 \pm 20.8(22.6$ to 36.3$)$ & $150.2 \pm 58.1(132.7$ to 167.6$)$ & $<0.001^{\mathrm{a}, \mathrm{b}}$ \\
RI & $0.7 \pm 0.1(0.7$ to 0.7$)$ & $0.7 \pm 0.1(0.7$ to 0.7$)$ & $0.13^{\mathrm{c}}$ \\
PSV & $41.4 \pm 14.3(36.7$ to 46.1$)$ & $42.2 \pm 15.3(37.6$ to 46.8$)$ & $0.63^{\mathrm{a}}$ \\
EDV & $12.4 \pm 5.6(10.1$ to 12.9$)$ & $11.5 \pm 4.6(10.7$ to 14.1$)$ & $0.63^{\mathrm{a}}$ \\
PVA & $29.0 \pm 10.2(25.7$ to 32.4$)$ & $30.7 \pm 12.0(27.0$ to 34.3$)$ & $0.50^{\mathrm{a}}$
\end{tabular}

${ }^{\text {aP }}$-value determined by non-parametric Mann-Whitney U-test. ${ }^{b}$ Indicates statistically significant values. ${ }^{c}$ Variable followed a normal distribution according to the Shapiro-Wilk test for normality and P-value was determined using the t-test. Values are expressed as the mean \pm standard deviation (95\% CI) or n. OSAHS, obstructive sleep apnea-hypopnea syndrome; AHI, apnea-hypopnea index; RDI, respiratory disturbance index; RI, resistivity index; PSV, peak systolic blood velocity; EDV, end diastolic blood velocity; PVA, patient-ventilator asynchrony.

$A H I$ and RDI. The AHI and RDI were significantly increased in the severe OSAHS cases vs. controls $(\mathrm{P}<0.001$; Table II). Furthermore, no association was obtained between the AHI and RI of the ophthalmic artery as detected by CDI $(\mathrm{P}=0.24)$.

$O P A$. No statistically significant difference was noted in the OPA measured using a Pascal tonometer between the two groups $(\mathrm{P}=0.59)$. Furthermore, no significant association was identified between OPA and the mean GCC or RI of the ophthalmic artery after CDI between the subject groups (Table I).

$R I$. No significant correlation between the mean average RNFL and RI was obtained in the control group. By contrast, in patients with severe OSAHS, the average RNFL was determined to decrease significantly with increments in $\mathrm{RI}(\mathrm{P}=0.0011)$, with a strong correlation coefficient of -68.2 (Fig. 1).

A similar effect was observed in the subgroups of mean superior and inferior RNFL. The mean superior RNFL values analyzed with regards to the RI demonstrated no statistically significant effect for the control group, but a significant decrease in the RNFL was noted with the increase of the $\mathrm{RI}$ in the severe OSAHS group and a strong correlation was obtained for this hypothesis $(\mathrm{P}=0.003$; correlation coefficient, -67.6; Fig. 2). For inferior RNFL values in relation to the RI, no statistical significance was noted for the control group, but in the OSAHS group, the RNFL appeared to decrease as the RI increased ( $\mathrm{P}=0.0017$; correlation coefficient, -69.5; Fig. 3).

When the RI was examined in relation to the average GCC, the latter appeared to be significantly decreased with RI increments in both groups (Fig. 4). For the control group the correlation coefficient was $-42.0(\mathrm{P}=0.008$ and for the OSAHS group, it was $-49.6(\mathrm{P}=0.0014)$.

Sleep position. With regards to differences in the RI between the eyes, no statistical significance was observed in the control group between the subjects that prefer to sleep 

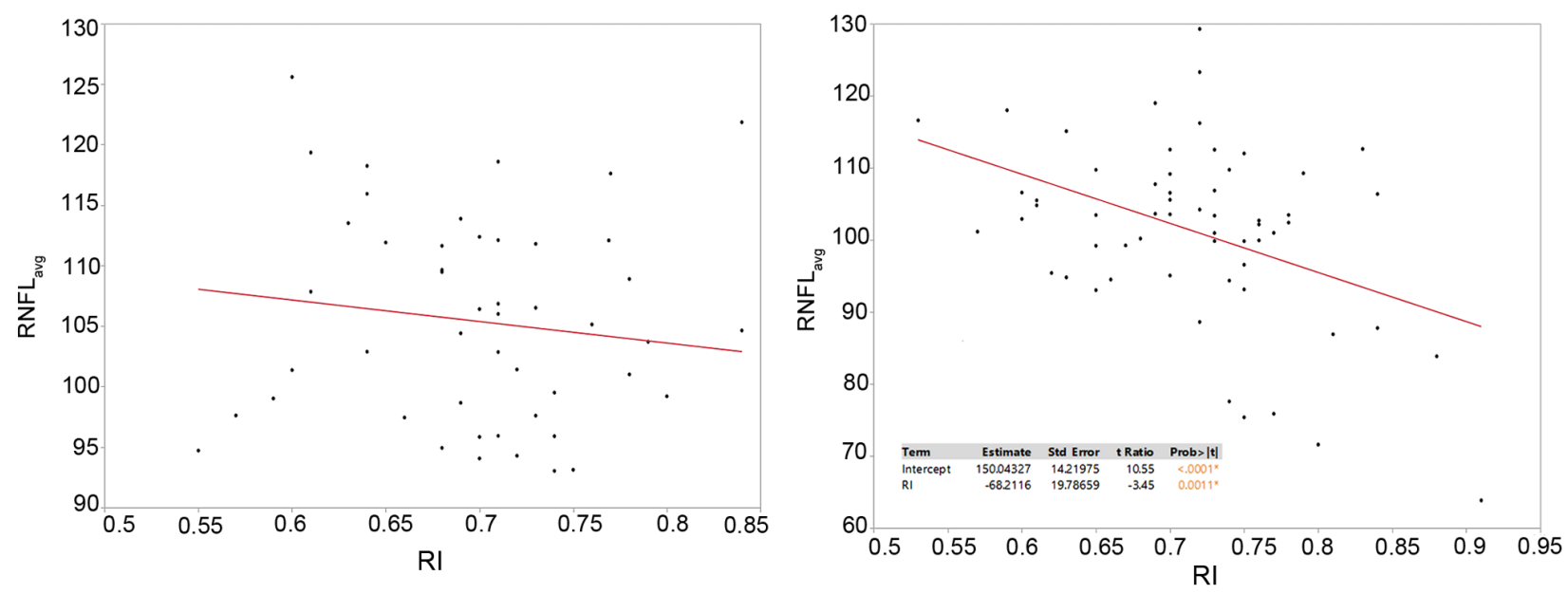

Figure 1. Average RNFL thickness in relation to RI increases in the control (left) and obstructive sleep apnea/hypopnea syndrome group (right). r=-68.21 \pm 19.78 $(\mathrm{P}=0.0011)$. RNFL, retinal nerve fiber layer; RI, resistivity index; avg, average; Std, standard; Prob, P-value.
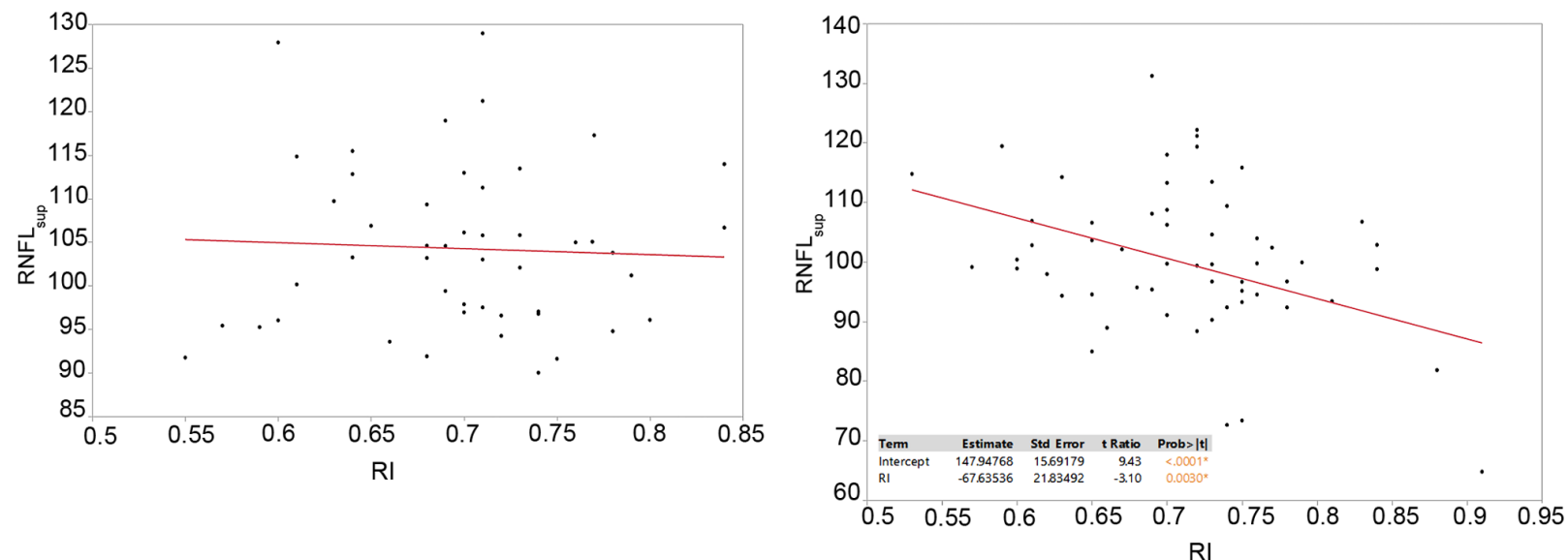

Figure 2. Superior RNFL thickness in relation to RI increases in the control (left) and obstructive sleep apnea/hypopnea syndrome group (right). r=-67.63 \pm 21.83 ( $\mathrm{P}=0.003)$. RNFL, retinal nerve fiber layer; RI, resistivity index; sup, superior; Std, standard; Prob, P-value.
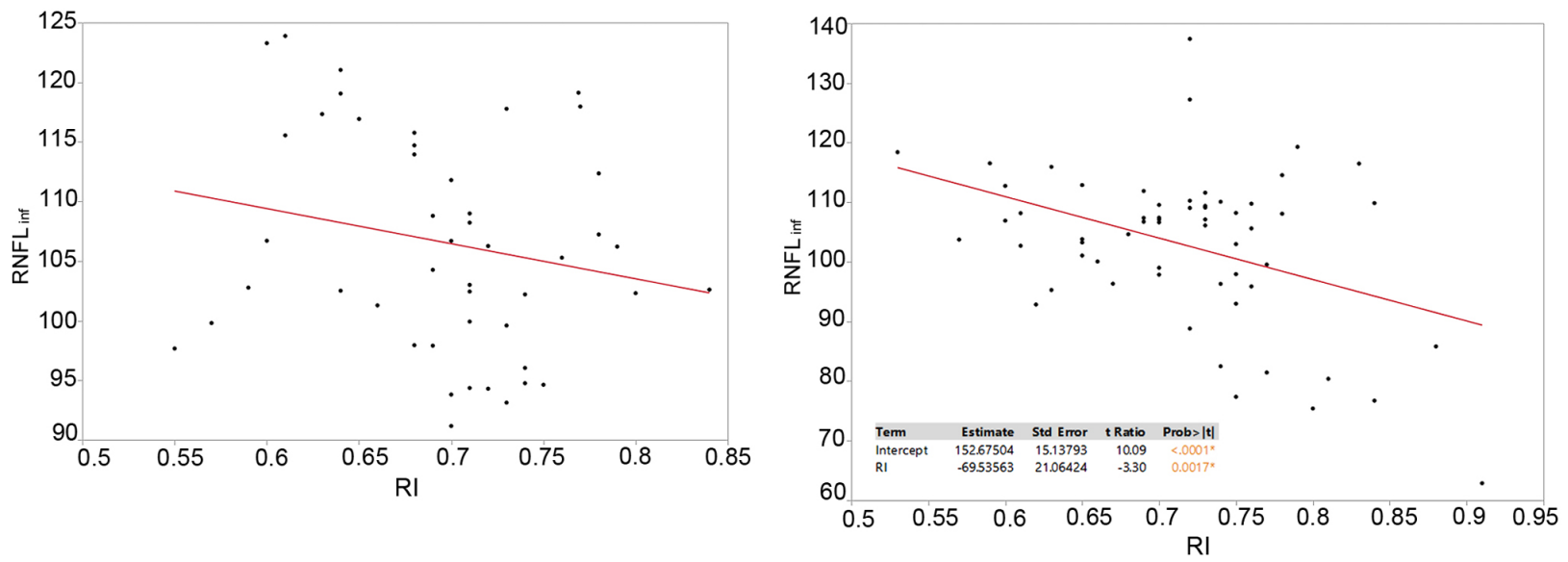

Figure 3. Inferior RNFL thickness in relation to RI increases in control (left) and obstructive sleep apnea/hypopnea syndrome group (right). $\mathrm{r}=-69.53 \pm 21.06$ $(\mathrm{P}=0.0017)$. RNFL, retinal nerve fiber layer; RI, resistivity index; inf, inferior; Std, standard; Prob, P-value.

on their side and those that sleep in the supine position $(\mathrm{P}=0.09)$. The same was observed for the OSAHS group $(\mathrm{P}=0.09)$. No significant association was identified between sleep position (left, right or supine) and RNFL or GCC difference between the two eyes in both groups $(\mathrm{P}=0.59$ and 0.91 , respectively). 

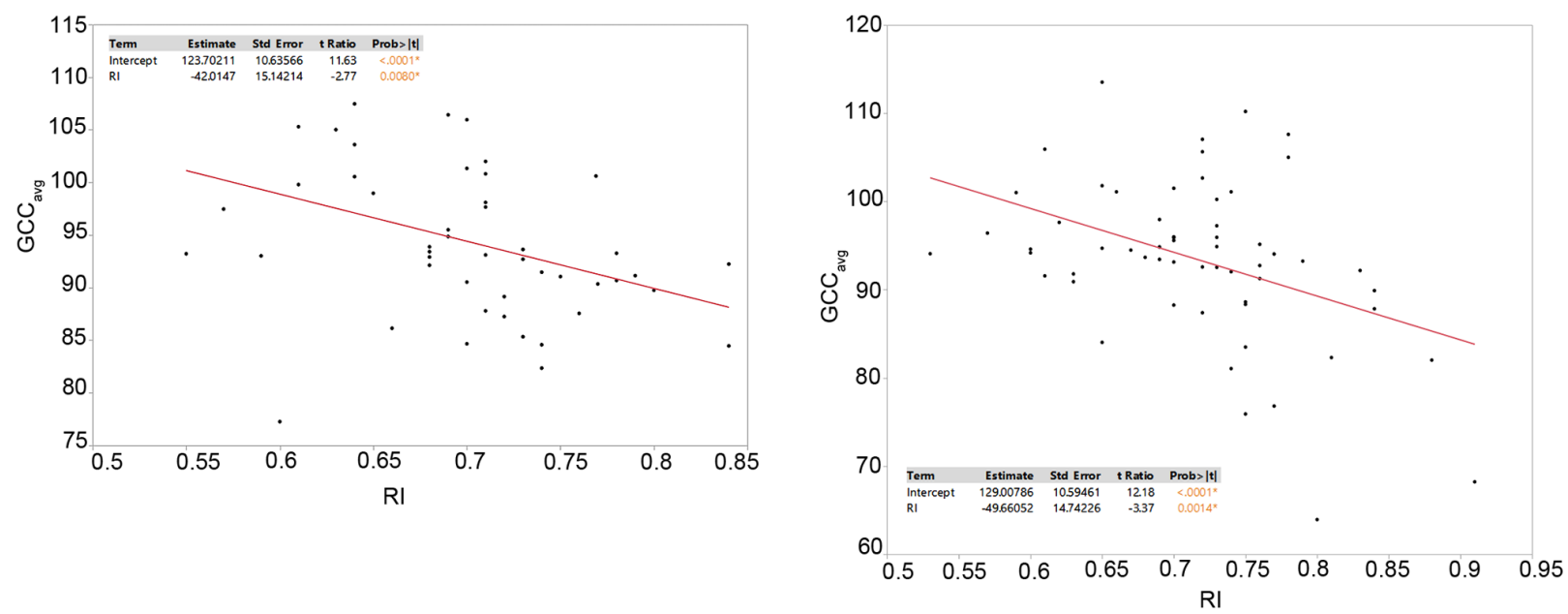

Figure 4. Average GCC thickness in relation to RI increases in the control (left) and obstructive sleep apnea/hypopnea syndrome group (right). $\mathrm{r}=-49.66 \pm 14.74$ $(\mathrm{P}=0.0014)$. RNFL, retinal nerve fiber layer; RI, GCC, ganglion cell complex, resistivity index; avg, average; Std, standard; Prob, P-value.

Saturated $\mathrm{O}_{2} \%$. A statistically significant decrement of near $1 \%$ was noticed in saturated blood oxygen percentage for the OSAHS group when compared with the control group $(\mathrm{P}=0.02)$ (Table II).

\section{Discussion}

It has been reported that OSAHS is associated with the pathogenesis of GON via both vascular and mechanical pathways $(10,16)$. An impaired blood flow to the optic nerve appears to be the most probable mechanism, with optic nerve fibres being directly damaged by an unstable oxygen concentration and reperfusion, followed by oxidative stress possessing an indirect effect (16). While certain studies observed a prevalence of GON ranging from 3-27\% in patients with OSAHS, others reported a minimal or no correlation (17-20). When patients with glaucoma were evaluated for OSAHS, previous studies indicated a prevalence ranging between 40 and $54 \%$, and it was particularly elevated in patients with normal tension glaucoma (18). Thus, this lack of consistency between findings means that the exact relationship between OSAHS and GON remains undetermined. Therefore, the present prospective, observational case-control study aimed to investigate the basis of this association by analysing the parameters of ocular blood flow.

Several studies have identified a positive association between IOP and OSAHS, including a study by Lin et al (19) revealing increased IOP values according to the severity of OSAHS (18-20). However, other studies indicated no significant differences in the mean IOP of patients with OSAHS compared with that of controls when measuring the IOP with various tonometry methods $(18,21)$. In the present study, no significant difference in the mean IOP was observed between controls and severe OSAHS cases either by Goldmann applanation tonometry or DCT. To the best of our knowledge, the present study was the first to obtain IOP values with both methods. Goldmann applanation tonometry is used in most studies, with the exception of the study by Lin et al (19), who used Perkins applanation tonometry, and that by Aşker et al (21), who used an ocular blood flow analyzer. When IOP measurements performed by either method are compared, a significant difference is observed for controls and subjects with OSAHS between the two tonometry methods $(\mathrm{P}<0.0001)$. Although studies have reported that the IOP measured using applanation and DCT exhibited no statistically significant difference, higher IOP values have been observed using DCT vs. applanation, with certain reports suggesting that this is observed in patients with low CCT values (22). Based on the aforementioned information and taking into consideration that CCT values in patients of the present study exhibited no statistical differences between groups $(\mathrm{P}=0.22)$, it was possible to attribute these present results to the small sample size. Recently, Arriola-Villalobos et al (23), in a study on 25 patients with severe OSAHS examining corneal parameters, determined significant differences in corneal elevation, corneal volume and minimum radius in patients with OSAHS compared with those in controls. This may provide a different explanation for the deviation in IOP between the two tonometry methods observed in the present study. Thus, it was concluded that further investigations with a larger sample are required in order to further signify these results.

With regard to the correlation between the AHI and GON parameters, a small number of studies have reported a statistical significance for IOP, whilst as per its relationship to mean defects in visual fields, results are varied in the existing studies (18). In the present study, a statistically significant difference was determined for both AHI and RDI in the cases of severe OSAHS compared with controls, with $\mathrm{P}<0.001$.

$\mathrm{RI}$ has been used as a marker of vascular resistance, which is altered by various pathologies that affect the vessel walls. Atherosclerosis, hypercholesterolemia or smoking, as well as alterations in blood pressure, nocturnal dipping and vasoconstriction, carotid artery disease and diabetes mellitus are among the pathologies reported in the current literature $(16,21,24-27)$. Such conditions were considered exclusion criteria for the present study in order to minimize the effect of vascular pathologies on ocular blood flow. Various studies have investigated the possible difference in RI between cases of OSAHS and controls, but these have reported no statistically significant difference (24-27). In the present study, a statistical 
significance was identified in the RI between the two groups $(\mathrm{P}=0.04)$.

The role of RI has been investigated in several studies examining OSAHS, mainly regarding its relationship with visual fields, which revealed altered indices in patients with OSAHS who exhibit no evidence of $\operatorname{GON}(18,20)$. Furthermore, an increased RI of the ophthalmic artery has been reported in patients with glaucoma compared with that in healthy subjects and has been suggested as a predictive factor for visual field progression in GON $(25,26)$. In the present study, RNFL thickness was indicated to decrease significantly with increased RI in patients with severe OSAHS but not for the control group. This was confirmed for average $(\mathrm{P}=0.0011)$, superior $(\mathrm{P}=0.0030)$ and inferior $(\mathrm{P}=0.0017) \mathrm{RNFL}$. ANOVA demonstrated that for every unit increase in the RI, a decrease of the RNFL by almost 68 units was obtained when analyzing the average and superior RNFL, and a decrease by decrease 69 units for the inferior RNFL for patients with OSAHS. Of note, when comparing RNFL values between the two groups, no significant difference was obtained in the subgroups of average, superior or inferior RNFL. This result suggests a complex effect of the RI on RNFL in OSAHS. While the effect of OSAHS on RNFL and GCC, measured either by OCT or scanning laser polarimetry, has been the focus of several studies, which have identified a severity relationship between RNFL thinning and OSAHS, other studies have indicated no correlation (18-20), and to the best of our knowledge, the present study was the first to associate the RI directly with RNFL.

Of note, evidence from the present study indicated a relationship between the RI and average GCC. Both the controls and patients with OSAHS exhibited decreased GCC values as the RI increased. In order to explain this result, no evidence regarding the effect of RI on ganglion cells was found in the current literature. Given that the RI may be indicative of decreased blood flow and decreased perfusion $(25,27)$, this result may suggest a particular vulnerability of macular Ganglion cells to altered blood flow, which may be possibly higher in patients with OSAHS.

Given these results, an examination of other CDI components, specifically PSV and EDV, may provide additional information and/or reveal more complex relationships.

The main limitation of the present study was the sample size. Another possible limitation was that all patients with OSAHS had severe disease (AHI >30), and thus, these results should be investigated in cases with milder disease.

In conclusion, a decrease in RNFL thickness that depends on RI increases in cases of severe OSAHS suggested a vulnerability of the RNFL in OSAHS. The decrease in GCC thickness as the RI increased in all subjects indicated that impaired perfusion, particularly in OSAHS, leads to structural changes. Thus, the long-term follow-up of patients with OSAHS should include OCT evaluation for changes in RNFL and GCC thickness, as well as CDI evaluation. Furthermore, patients with increased ophthalmic artery RIs on CDI, regardless of the etiology, should be monitored for GCC changes.

\section{Acknowledgements}

Not applicable.

\section{Funding}

No funding was received.

\section{Availability of data and materials}

The datasets used and/or analyzed during the current study are available from the corresponding author on reasonable request.

\section{Authors' contributions}

GD, AT, AS, CT, ETD, VK and GL conceived and designed the study. GD, AL, AS performed a literature search. GD, AT and $\mathrm{CT}$ acquired the data and confirmed the authenticity of the raw data. GD, AT and AL analyzed and interpreted the data. GD, AT, AL and CT performed critical analysis and review of the literature. GD, AT and AL drafted the manuscript. CT, ETD, VK and GL critically revised the article for important intellectual content. All authors read and approved the final manuscript.

\section{Ethics approval and consent to participate}

Informed consent was obtained from all patients prior to surgery and all data were collected according to the principles of The Declaration of Helsinki. The study was approved by Democritus University of Thrace, School of Medicine (approval no. 20721/1503) and the Ethics Committee of Konstantopouleio-Patission General Hospital (approval no. 233/25.6.15).

\section{Patient consent for publication}

Not applicable.

\section{Competing interests}

The authors declare that they have no competing interests.

\section{References}

1. Ryan CM and Bradley TD: Pathogenesis of obstructive sleep apnea. J Appl Physiol (1985) 99: 2440-2450, 2005.

2. Mojon DS, Hess CW, Goldblum D, Böhnke M, Körner F and Mathis J: Primary open-angle glaucoma is associated with sleep apnea syndrome. Ophthalmologica 214: 115-118, 2000.

3. Mojon DS, Hess CW, Goldblum D, Fleischhauer J, Koerner F, Bassetti C and Mathis J: High prevalence of glaucoma in patients with sleep apnea syndrome. Ophthalmology 106: 1009-1012, 1999.

4. McNab AA: The eye and sleep apnea. Sleep Med Rev 11: 269-276, 2007.

5. Marcus DM, Costarides AP, Gokhale P, Papastergiou G, Miller JJ, Johnson MH and Chaudhary BA: Sleep disorders: A risk factor for normal tension glaucoma? J Glaucoma 10: 177-183, 2001.

6. Weinreb RN and Khaw PT: Primary open-angle glaucoma. Lancet 363: 1711-1720, 2004.

7. Onen SH, Mouriaux F, Berramdane L, Dascotte JC, Kulik JF and Rouland JF: High prevalence of sleep-disordered breathing in patients with primary open-angle glaucoma. Acta Ophthalmol Scand 78: 638-641, 2000.

8. Klemm M and Gesser C: The relevance of diabetes for patients with glaucoma. Klin Monbl Augenheilkd 231: 116-120, 2014 (In German). 
9. Holló G, Cvenkel B, Teus MA, Irkec MT, Astakhov YS, Chiselita D, Petkova N, Liehneová I, Kaluzny BJ, Kóthy P, et al: Is there any difference in target intraocular pressure for exfoliative glaucoma patients with cardiovascular disease history? Eur J Ophthalmol 20: 1000-1006, 2010.

10. Mojon DS, Hess CW, Goldblum D, Boehnke M, Koerner F, Gugger M, Bassetti C and Mathis J: Normal-tension glaucoma is associated with sleep apnea syndrome. Ophthalmologica 216 : $180-184,2002$

11. Kremmer S, Selbach JM, Ayertey HD and Steuhl KP: Normal tension glaucoma, sleep apnea syndrome and nasal continuous positive airway pressure therapy-case report with a review of literature. Klin Monbl Augenheilkd 218: 263-268, 2001 (In German).

12. Khandgave TP, Puthran N, Ingole AB and Nicholson AD: The assessment of sleep apnoea as a risk factor in glaucoma. J Clin Diagn Res 7: 1391-1393, 2013.

13. Bendel RE, Kaplan J, Heckman M, Fredrickson PA and Lin SC: Prevalence of glaucoma in patients with obstructive sleep apnoea-a cross-sectional case-series. Eye (Lond) 22: 1105-1109, 2008.

14. Lin CC, Hu CC, Ho JD, Chiu HW and Lin HC: Obstructive sleep apnea and increased risk of glaucoma: A population-based matched-cohort study. Ophthalmology 120: 1559-1564, 2013.

15. McNicholas WT: Diagnosis of obstructive sleep apnoea in adults. Proc Am Thorac Soc 5: 154-160, 2008.

16. Trivli A, Koliarakis I, Terzidou C, Goulielmos GN, Siganos CS, Spandidos DA, Dalianis G and Detorakis ET: Normal-tension glaucoma: Pathogenesis and genetics. Exp Ther Med 17: 563-574, 2019.

17. Swaminathan SS, Bhakta AS, Shi W, Feuer WJ, Abreu AR, Chediak AD and Greenfield DS: Is obstructive sleep apnea associated with progressive glaucomatous optic neuropathy? J Glaucoma 27: 1-6, 2018.

18. Pérez-Rico C, Gutiérrez-Díaz E, Mencía-Gutiérrez E, Díaz-de-Atauri MJ and Blanco R: Obstructive sleep apnea-hypopnea syndrome (OSAHS) and glaucomatous optic neuropathy. Graefes Arch Clin Exp Ophthalmol 252: 1345-1357, 2014.

19. Lin PW, Friedman M, Lin HC, Chang HW, Pulver TM and $\mathrm{Chin} \mathrm{CH}$ : Decreased retinal nerve fiber layer thickness in patient with obstructive sleep apnea/hypopnea syndrome. Graefes Arch Clin Exp Ophthalmol 249: 585-593, 2011.
20. Huseyinoglu N, Ekinci M, Ozben S, Buyukuysal C, Kale MY and Sanivar HS: Optic disc and retinal nerve fiber layer parameters as indicators of neurodegenerative brain changes in patients with obstructive sleep apnea syndrome. Sleep Breath 18: 95-102, 2014.

21. Assker S, Timucin OB, Ursavas A, Aslanci ME, Baykara M, Erturk H, Asker M, Yilmaz S and Kaya DT: Obstructive sleep apnea syndrome and blood flow to the eyes. East J Med 18: $165-171,2013$.

22. Willekens K, Rocha R, Van Keer K, Vandewalle E, Abegão Pinto L, Stalmans I and Marques-Neves C: Review on dynamic contour tonometry and ocular pulse amplitude. Ophthalmic Res 55: 91-98, 2015.

23. Arriola-Villalobos P, Benito-Pascual B, Peraza-Nieves J, Perucho-González L, Sastre-Ibañez M, Dupré-Peláez MG, Asorey-García A and Fernández-Sánchez-Alarcos JM: Corneal topographic, anatomic, and biomechanical properties in severe obstructive sleep apnea-hypopnea syndrome. Cornea 39: 88-91, 2020.

24. Erdem CZ, Altin R, Erdem LO, Kargi S, Kart L, Cinar F and Ayoglu F: Doppler measurement of blood flow velocities in extraocular orbital vessels in patients with obstructive sleep apnea syndrome. J Clin Ultrasound 31: 250-257, 2003.

25. Martínez A and Sánchez M: Predictive value of colour Doppler imaging in a prospective study of visual field progression in primary open-angle glaucoma. Acta Ophthalmol Scand 83: 716-722, 2005.

26. Magureanu M, Stanila A, Bunescu LV and Armeanu C: Color Doppler imaging of the retrobulbar circulation in progressive glaucoma optic neuropathy. Rom J Ophthalmol 60: 237-248, 2016.

27. Galassi F, Sodi A, Ucci F, Renieri G, Pieri B and Baccini M: Ocular hemodynamics and glaucoma prognosis: A color Doppler imaging study. Arch Ophthalmol 121: 1711-1715, 2003.

This work is licensed under a Creative Commons Attribution-NonCommercial-NoDerivatives 4.0 International (CC BY-NC-ND 4.0) License. 